\title{
GCU
}

Glasgow Caledonian

University

University for the Common Good

\section{Rolling stock door system reliability improvement using maintenance optimisation}

Alkali, B.M.; Orsi , V.; Ramani, A.

Published in:

Proceedings of the Third International Conference on Railway Technology: Research, Development and Maintenance

DOI:

$10.4203 /$ ccp. 110.281

Publication date:

2016

Document Version

Author accepted manuscript

Link to publication in ResearchOnline

Citation for published version (Harvard):

Alkali, BM, Orsi , V \& Ramani, A 2016, Rolling stock door system reliability improvement using maintenance optimisation. in J Pombo (ed.), Proceedings of the Third International Conference on Railway Technology: Research, Development and Maintenance. vol. 110, 281, Civil-Comp Press, 3rd International Conference on Railway Technology: Research, Development and Maintenance, Cagliari, Italy, 5/04/16.

https://doi.org/10.4203/ccp.110.281

\section{General rights}

Copyright and moral rights for the publications made accessible in the public portal are retained by the authors and/or other copyright owners and it is a condition of accessing publications that users recognise and abide by the legal requirements associated with these rights.

Take down policy

If you believe that this document breaches copyright please view our takedown policy at https://edshare.gcu.ac.uk/id/eprint/5179 for details of how to contact us. 
Rolling Stock Door system Reliability Improvement through Maintenance Optimisation

B.M Alkali ${ }^{1}$, V. Orsi ${ }^{2}$, and A. Ramani ${ }^{3}$

${ }^{1}$ Department of Engineering, Glasgow Caledonian University, United Kingdom

${ }^{2}$ Interfleetgroup, SNC Lavalin House, Derby, United Kingdom

${ }^{3}$ Abellio Scotrail Ltd, 36 Renfield Street, Glasgow, United Kingdom

\begin{abstract}
The maintenance of rolling stock is a major task because an in-service failure causes delays and passenger dissatisfaction. This paper focuses on the door system of the Class 158 Diesel Multiple Unit DMU train fleet. The door system consists of several functionally dependent components and is operated in-service continuously and is only monitored at discrete time points. The doors have a combination of serial/parallel systems consisting of over 100 components and the component lifetimes are Weibull distributed which implies that they are aging with time. A failure analysis of the door system is conducted using the reliability centred maintenance approach and an attempt is made to optimise the door maintenance practices given that they have been operating for certain number of periods. The door systems consist of several worn and aging components and are considered to be stochastically independent. Cost effective preventive maintenance optimisation is performed using specialist simulation software, which involves incorporating the average number of door components replaced over some finite horizon. A numerical assessment of the door components is conducted and a piecewise Deterministic Markov Process model is proposed in an attempt to optimise a preventive maintenance strategy of the doors.
\end{abstract}

Keywords: rolling stock, doors, maintenance optimisation, Markov process, availability, performance.

\title{
1 Introduction
}

The maintenance of rolling stock is a major task because an in-service failure could lead to serve delays and hence passenger dissatisfaction. Moreover, in-service failures increase maintenance cost and have a knock on effect on the overall fleet maintenance budget. A train operating company in Scotland, United Kingdom is 
keen to improve preventive and proactive maintenance of safety critical systems on its train fleets. The Class 158 Diesel Multiple Unit (DMU) train fleet is considered in this study. The annual railway calendar in Scotland is split into 13 four week periods. At the end of each period the performance data for each fleet is collated and analysed. The performance plans are then implemented on areas of poor performance to improve the affected system. The reliability and availability performance of the entire fleet is expected to be supported by a good preventive maintenance plan. This paper focuses on the door system of the Class 158 train fleet. The door system consists of several functionally dependent components. The door system is used continuously and monitored at discrete time points. This paper attempts to optimize the maintenance of the door system given that they have been operating for certain number of period. The nature of the door system component design weaknesses is not revealed in this paper because of confidentiality.

In order to ensure that the door systems are functioning properly an examination is undertaken as a form of preventive maintenance action. The door system is a combination of serial/parallel systems consisting of over 100 components. The door system component lifetime is Weibull distributed which implies that they are aging with time. There are a lot of aging models that have been used in maintenance optimizations of systems. The virtual age model presented in [1], states that a repair modifies the intensity function for a system's virtual age to some extent at each corrective maintenance action. For these repairs, the virtual age at any given time is determined by a variety of additive age-reduction factors. The concept of age reduction to model failure event data of systems subject to periodic preventive maintenance and imperfect corrective maintenance is discussed in [2]. However, the age reduction model effectively resets a system to a younger age, which is only an approximation to a renewal process.

The virtual age model on the other hand has been applied to systems under a block replacement policy [3]. Although it can serve as a very good statistical model, a critical defect of the virtual age model and its many variants is that they do not provide a realistic description of the failure processes. A variant of the virtual age model is proposed in [4]. A first order Arithmetic Reduction of Age models and the failure rates that are intrinsically of the Weibull type is presented in [5]. In order to model the door system aging component, a piecewise deterministic Markov process in [6] is considered. In this paper a framework for the modelling is presented using the failure intensity of the door system.

\section{The Door System}

The class 158 DMU fleet is a two car carriage and the door system components are functionally dependent. Each of the class 158 train is fitted with four external biparting swing plug doors per vehicle for both passenger and crew access. The doors are electrically controlled and pneumatically operated. They are opened when operated by the train conductor operating the door key switch (DKS) on the door 
control panel and pressing both door open buttons together, this generates a door release signal for one side of the train. All external doors are double leaf, i.e. biparting and have obstruction features to prevent injury to persons trapped by a closing door. They have obstruction detection on both opening and closing cycles. Each external door can be isolated by means of a valve situated above the door which shuts-off the main air to the door. Assuming the safety criteria is met when a door release relay is initiated, this places the doors on the side of the train released under the control of the passenger through the local door open / close buttons illuminating.

When a passenger presses the illuminated door open button, the pneumatic system operates to allow air in to the torque cylinder. This unlocks the door over centre locking mechanism and causes the torque cylinder to rotate. The torque cylinder operates linkages attached to each door leaf opening the door. When a passenger presses the illuminated close button or the conductor presses the close button on the guard's door control panel, the torque cylinder operates to close the open door. The door closes and is mechanically locked over centre.

\subsection{Door Reliability Performance}

The door performance is measured at various intervals - daily on depot, weekly at functional level and four weekly for external reporting. The DMU fleet performance is calculated by analysing the fleet in-service failures. Trains that fail in-service can have different degree of disruption to services for example, minute's delayed, part cancellation of a service or full cancellation of a service. This is attributable to the train maintenance procedure and thus affects the train performance. In order to analyse the train reliability various calculations are made. The industry standard for reliability is classed as Miles Per Technical Incident (MTIN). The MTIN indicates how far a train has travelled between technical incidents (i.e. maintenance related defects), which corresponds to the Mean Time Between Failure (MTBF) used in other industries. The passenger door system is selected for analysis in this paper based on how the system has been affected in service, prioritization set by occurrence, and minutes delay. A performance summary for 12 months is presented in Figure 1 below. A 3 period moving average trend line is fitted to the door incidents, and it is observed that the door failure incidents are increasing with time.

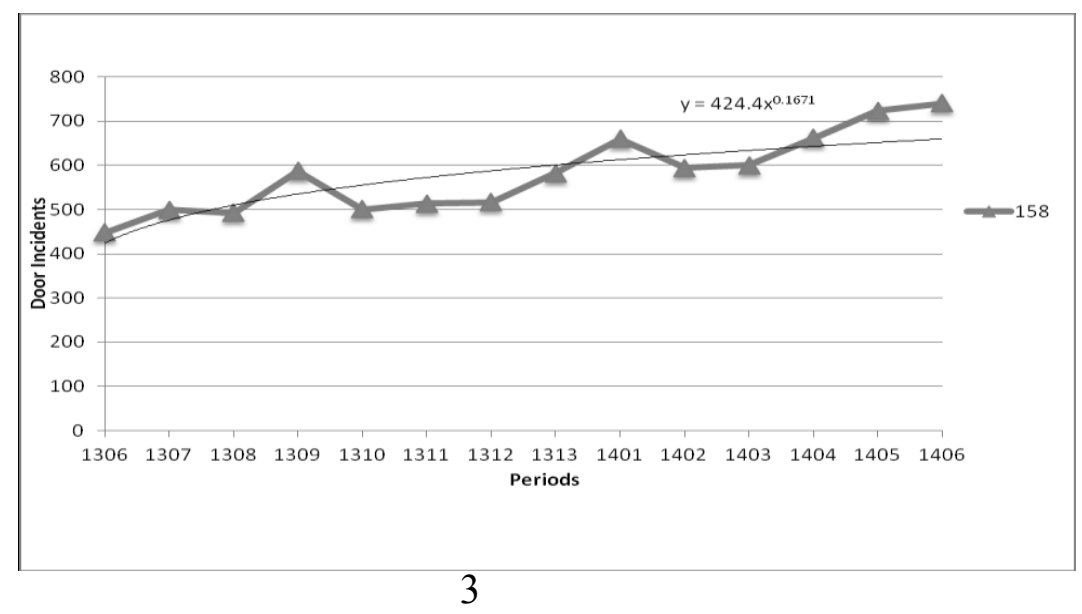


Figure 1. Class 158 fleet door performance summary

The class 158 door systems has the most technical incidents and minutes attributed to it against the other safety critical systems on the class 158 DMU fleet and this due to a combination of factors primarily including the complexity of the system with integration of electrical, mechanical and pneumatic components; the inherent unreliability of aged components; and difficulty in bringing the unit back for maintenance due to geographical and logistical constraints.

\subsection{Door Maintenance Schedule}

The current maintenance strategy is based around details obtained from the OEM of the individual system. The maintenance schedule of the door systems incorporates an exam every 20,000 miles, and the rail industry maintenance is based solely on distance covered rather than operating time. The maintenance activity cycles from basic cleaning tasks every 10,000 miles to in-depth component changes and rebuilds every 700,000 miles. The schedule is broken into two categories:

- Vehicle Maintenance Schedule, VMS, lists all maintenance tasks and a periodicity when the specific task should be carried out.

- Maintenance Star Chart lists all maintenance tasks and allocates them to specific exams.

In total there are over 160 individual tasks within the Vehicle Maintenance Schedule of which there are 6 scheduled maintenance procedures that are specific to the passenger door system. Taking into account that the fleet is expected to run an expected 100,000 miles per year then the amount of maintenance activity which is scheduled to be performed on the door system has to be suitably split as it is not operationally feasible to undertake all tasks at one large annual exam only.

\section{Maintenance of Door Systems}

The door systems have multiple components and the components are functionally dependent. We assume that if one or two components are in a fail state then a door failure is initiated. The failure can be attributed by a particular failure mode. For example, component $\mathrm{A}$ and $\mathrm{B}$ can be in failed state due to failure mode $\mathrm{F}_{1}$ and $F_{2}$ respectively. $F_{1}$ failure modes on component $\mathrm{A}$ may fail and the systems could be working fine conversely when a component fails in $F_{2}$ failure mode the door system does not function regardless of the state of the other component. Such failure could lead to a delay, part cancellation or full cancellation of train service. A corrective maintenance or preventive maintenance actions can be undertaken to replace or repair the component instantly.

\subsection{Maintenance strategy}


Preventive maintenance is a schedule of planned maintenance actions aimed at the prevention of breakdowns and failures. Preventive maintenance activities include systems checks, partial or complete overhauls at specified periods, oil changes and lubrication. In addition, workers can record equipment deterioration so they know to replace or repair worn parts before they cause system failure and recent technological advances in tools for inspection and diagnosis have enabled even more accurate and effective equipment maintenance. The ideal preventive maintenance program would prevent all system component failure before it occurs.

On the other hand corrective maintenance task is performed to identify, isolate, and rectify a fault so that the failed equipment, machine, or system can be restored to an operational condition within the tolerances or limits established for in-service operations. An opportunistic maintenance can be conducted at the time of corrective maintenance if the time widow allows for an opportunity to be utilised.

Maintenance intervention involves preventive maintenance and corrective maintenance (unplanned, CM). Preventive maintenance has been described as the most difficult activity to model in the field of maintenance [7, 8]. The complexity of modelling PM stems from the difficulty in quantifying the effects of performing PM at different intervals [9]. The main outstanding problem here is to choose which maintenance policy is best as a routine to be undertaken for improving the door components, and hence system, utilisation at the lowest expected cost.

\section{Maintenance Modelling}

The corrective maintenance and preventive maintenance are conducted instantaneously because there duration of a maintenance action on the door is negligible compared to the lifetime of the component. In order to quantify the effect of the preventive maintenance actions on the door component lifetimes, one need to identify the failure modes related to the door failure. The choice of which failure mode to consider depends upon the history of the failure process and this could help to support the standard model selection procedures. The arithmetic age reduction model ARA $A_{1}$ proposed in [5] is considered here and failure intensity is defined by the expression in equation (1) below.

$$
\lambda_{t}=\lambda_{\theta}\left(t-\rho T N_{t}\right)
$$

where $\lambda_{\theta}$ the intrinsic failure rate of the unmaintained component and $\theta$ is the parameter estimator. Defining the events as PM or CM actions, suppose we observe actual event times $t_{k}$ in the interval $(0, t]$, with corresponding censoring indicator variables $\rho_{k}$ where

$$
\rho_{k}= \begin{cases}0 ; & \text { no failure at timet } \\ k & (\mathrm{PM}) \\ 1 ; & \text { failure at timet } \\ k & (\mathrm{CM})\end{cases}
$$


for $k=1,2, \ldots \ldots, n$ where $n=N_{t}$. The corresponding likelihood function is given by

$$
L\left\{\lambda_{t} ; H(t)\right\} \propto\left\{\prod_{k=1}^{n} \lambda_{\theta}\left(t_{k}^{-}-T^{k} N_{t}\right)^{\rho_{k}}\right\} \exp \left\{-\int_{0}^{t} \lambda_{\theta}\left(t_{k}-T N_{t}\right) d t\right\}
$$

where $H(t)$ represents the history of the failures and maintenance actions. The appropriate failure rate considering the aging process is of Weibull type and it is given as

$$
\lambda_{\theta}(t)=\frac{\beta}{\alpha}\left(\frac{t}{\alpha}\right)^{\beta-1}
$$

with $\theta=(\alpha, \beta)$ and the intensity function of an arithmetic age reduction is given as

$$
\lambda_{t}=\frac{\beta}{\alpha}\left(\frac{t-\rho T N_{t^{-}}}{\alpha}\right)^{\beta-1}
$$

In order to estimate the parameters the maximum likelihood methods can be used and the log-likelihood is given as

$$
l\left\{\lambda_{t} ; H(t)\right\} \& \sum_{k=1}^{n} \rho_{k} \log \lambda_{\theta}\left(t_{k}^{-}-\log T^{k} N_{t}\right)-\int_{0}^{t} \lambda_{\theta}\left(t_{k}-T N_{t}\right) d t
$$

where the symbol $\&$ is used to represent equality subject to an additive constant. The maximum likelihood estimates may be found from the above log-likelihood by partial differentiation and solution of the resulting likelihood equations. The MLE is maximized numerically through simulation. The result is to be used to model the door systems within the framework of the PDMP and this is presented in Section 4.1 .

\subsection{Piecewise Deterministic Markov Process}

The PDMP is introduced by Davis in 1984 [6] and modeling of this type has been used for nuclear power generating plant [10]. A PDMP is a hybrid process such that $\left(I_{t} ; X_{t}\right) t \geq 0$, the first component $I_{t}$ is considered to be discrete, with values in a finite state space $E$. For example two-component system can be modelled with a PDMP with state space $\mathrm{E}=\{(1 ; 1) ;(1 ; 0) ;(0 ; 1)\}$ (1 for up and 0 for down). The state $(0 ; 0)$ is never reached because maintenance actions are instantaneous, so that the system runs continuously. The application of a maintenance optimisation of two components systems using the PDMP techniques is demonstrated in [11]. In this paper we presented a framework that could link opportunistic maintenance as a form of preventive maintenance.

In order to identify each component failure mode, we define the probabilities $p_{F_{i}}^{A}$ and $p_{F_{i}}^{B}$ with $i \in[1,2]$ where $p_{F_{i}}^{A}$ is the probability that component $A$ fails in mode $F$ i given that component $\mathrm{B}$ is not in a fail state and still operating. Therefore

$$
p_{F_{i}}^{A}+p_{F_{i}}^{A}=1
$$


When the two components are working, failure of component $A$ in mode $F_{1}$ does not cause the door to fail and hence initiate an in-service failure. If a failure is not detected component $A$ is not replaced. The PDMP jumps from state $(1 ; 1)$ to state $(0$; 1). The PDMP transition kernel is given as:

$$
b\left(\left((1,1),\left(x_{A}, x_{B}, t\right)\right),\left((0,1),\left(d y_{A}, d y_{B}, d s\right)\right)\right)=p_{F_{1}}^{A} \cdot a_{A}\left(x_{A}, t\right) \rho_{x_{A}, x_{B}, t}\left(d y_{A}, d y_{B}, d s\right)
$$

Similarly, if component $B$ fails as a result of failure mode $F_{l}$, the systems will still be operating and no failure is detected. The PDMP transition kernel in this case is given as;

$$
b\left(\left((1,1),\left(x_{A}, x_{B}, t\right)\right),\left((1,0),\left(d y_{A}, d y_{B}, d s\right)\right)\right)=p_{F_{1}}^{B} \cdot a_{B}\left(x_{B}, t\right) \rho_{x_{A}, x_{B}, t}\left(d y_{A}, d y_{B}, d s\right)
$$

A preventive maintenance action is expected to be conducted when $t \in(1,2$, $\ldots, T)$ over the specified period of observation. So we define PDMP transition kernel during preventive maintenance for all $k \in(1,2, \ldots, T)$. In order to initialize the PDMP we define a function to support the empirical law of entry schedule for preventive maintenance when the system is working $(t=0)$, and we assume that the systems are in state $(1,1)$. The application of this concept is under development and still on-going.

\section{Maintenance Optimisation}

Maintenance optimisation is essential as the time period require for maintenance is negligible. A preventive maintenance strategy is already in place for the door systems but not cost effective. In addition to the current maintenance strategy we propose the use of opportunistic maintenance to replace components based on their state. To optimise the maintenance of the door system, the age is considered to minimize expected cost per unit time. An optimal cost function that minimizes the mean cost over time period $T$ can be assessed using the cost function

$$
C(T)=N_{u n} \cdot\left(C_{A}+C_{B}+C_{u n}\right)+N_{A}^{f} \cdot\left(C_{A}\right)+N_{B}^{f} \cdot\left(C_{B}\right)+N_{A}^{p m} \cdot\left(C_{A}\right)+N_{B}^{p m} \cdot\left(C_{B}\right)+N_{p m} \cdot\left(C_{p m}\right)
$$

Where $\left(C_{A}\right)$ is the cost of component A replacement, $\left(C_{B}\right)$ is the cost of component B replacement, $\left(C_{u n}\right)$ is the cost of unobserved random events, $\left(C_{p m}\right)$ is the cost of preventive maintenance. $\left(N_{A}^{f} \& N_{B}^{f}\right)$ is the number of $\mathrm{A}$ and B failures respectively. $\left(N_{A}^{p m} \& N_{B}^{p m}\right)$ is the number of preventive maintenance of $\mathrm{A}$ and $\mathrm{B}$ respectively and $\left(N_{p m}\right)$ is the number of preventive maintenance activity. We intend to use the expression within the framework of PDMP and simulate the algorithm to obtain the optimum preventive maintenance time schedule. The concept proposed in the paper is new and it applications to the railway transport industry is scarce.

\subsection{Two State Markov Process}

A numerical example of a two state transition Markov process of the failure of the door emergency egress handle and its related failure mode is conducted. The 
probability of failure is estimated based on the number of incidents leading to inservice failures as a result of its related failure modes. The working and failed state of the emergency egress handle is represented as " 0 " for working state and " 1 " for failed state. The transition state space probability $\operatorname{Pw}(\mathrm{t})$ and $\operatorname{Pf}(\mathrm{t})$ of the emergency egress handle is presented in the graph in Figure 2

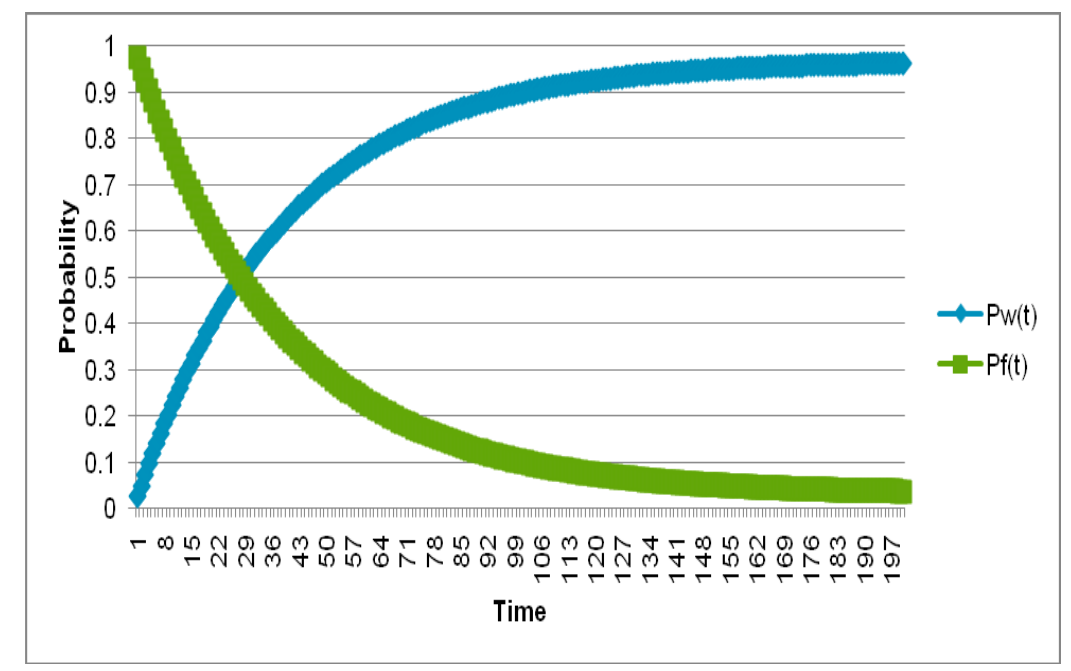

Figure 2. Emergency Egress handle two state transition probability

\section{Door RCM Analysis}

A comprehensive reliability centred maintenance analysis is conducted on the door system and over 356 failure modes are identified. Further analysis is conducted using a specialist simulation Isograph Availability Workbench RCMCost Software. The Availability Workbench Software uses the framework of the reliability centred maintenance approach. In order to optimize a maintenance regime it is essential to consider: mean time to failure, equipment used during maintenance, spares, labor, operational costs and the condition of equipment after repair. The Software offers recommendations regarding maintenance or spare part optimization and this depends on the lifetime and simulation options.

\subsection{Door Simulation Results}

This section highlights which door system component are subject to reoccurring failures and the costs associated with these failures include; corrective costs, inspection costs, equipment costs, spares costs and the costs due to delays and cancellations. The software is used to perform simulations and a global time of 10 year interval is specified. Multiple lifetime simulation is conducted to optimise the maintenance over a specified number of years. The result revealed that the expected estimated number of safety incidents predicted via the safety criticality shows that they are all approximately the same each year. This is due to the fact that the safety 
criticalities in the maintenance optimisation graphs are calculated based on the severity weighting and the number of failures. Therefore, the expected number of safety incidents is an approximation regardless of the lifetime. Similarly, the door operational criticality is calculated in the exact same way as the safety criticality and the failure rate is assumed to be constant. This implies that the number of failures in any lifetime would yield the same amount of failures in a year.

A series of graphs are generated from the simulations of the door historical maintenance and failure data. The results obtained show that the current maintenance is not cost effective as shown in Figure 3. The recommendations set out from the RCM Cost analysis recommend that maintenance intervals can be extended and significant cost savings can be achieved while maintaining current maintenance strategy to improve performance.

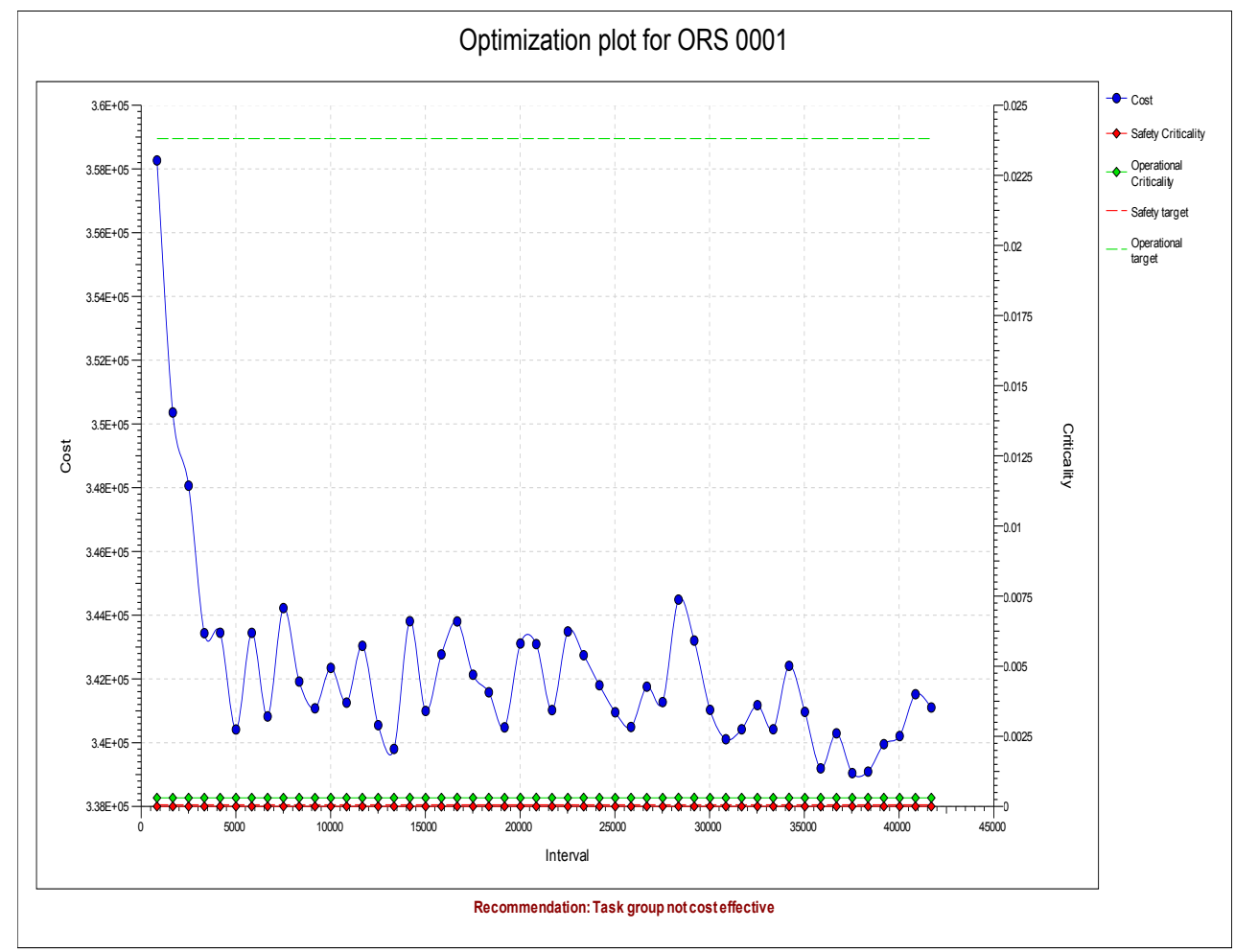

Figure 3 Maintenance optimisation simulation results

\subsection{Conclusion}

This paper present result from a comprehensive reliability centred maintenance analysis and it application to door system of Class 158 Diesel Multiple Unit train fleet. A Markov model is proposed that could be used to model failure patterns and its related failure modes. A numerical assessment of the door components is conducted and a piecewise Deterministic Markov Process model is proposed in an attempt to optimise a preventive maintenance strategy of the doors. The Availability Workbench RCM Cost analysis is conducted on the door systems and door is 
identified as a complex system with too many individual maintenance requirements. The RCM Cost analysis clarified the potential cost savings and safety criticality/ operational criticality of extending maintenance practices. Therefore, the maintenance intervals are only extendable if the associated safety critical system maintenance is improved. If safety critical system maintenance is reduced the amount of in-service incidents, then the maintenance can be extended in increments of 10,000 miles. At the moment all the known failures from the RCM Analysis are expected to manifest in service at some point in the future. Therefore, it is imperative to target maintenance at these known failures to reduce the amount of failures occurring in-service. The appropriate maintenance strategy must be investigated further to determine which is most suitable.

\section{Acknowledgement}

This project is supported by ScotRail under the knowledge transfer partnership (No. 9070) with Glasgow Caledonian University.

\section{References}

[1] M. Kijima. Some Results for Repairable Systems with General Repair. Journal of Applied Probability. 26: 89 - 102, 1989.

[2] N. Jack. Age-Reduction Models for Imperfect Maintenance. IMA Journal of Mathematics Applied in Business and Industry. 9 (4): 347 - 354, 1998.

[3] H. Wang, and H. Pham. Optimal Age-dependent Preventive Maintenance Policies with Imperfect Maintenance. International Journal of Reliability, Quality and Safety Engineering. 3 (2): 119 - 135, 1996.

[4] C. Dorado, M. Hollander, J. Sethuraman. Nonparametric Estimation of General Repair Models. Annals of Statistics: 25: 1140 - 1160, 1997.

[5] L. Doyen, and O. Gaudoin. Classes of Imperfect Repair Models based on Reduction of Failure Intensity or Virtual Age. Reliability Engineering and System Safety. 84: 45 - 56, 2004.

[6] M. Davis. Piecewise Deterministic Markov Processes: a general class of nondiffusion stochastic models. Journal of Royal Statistical Soc. (B) 46, 353-388, 1984.

[7] A.H. Christer, and W.M Waller. An Operational Research Approach to Planned Maintenance: Modelling PM for a Vehicle Fleet. Journal of the Operational Research Society. 35: 967 - 984, 1971.

[8] K.A.H Kobbacy, B.B Fawzi, D.F Percy, and H.E Ascher. A Full History Proportional Hazards Model for PM Scheduling. Quality and Reliability Engineering International. 13: 187 - 198, 1997.

[9] D.F Percy, K.A.H Kobbacy, and B.B Fawzi. Setting Preventive Maintenance when Data are Sparse. International Journal of Production Economics. 51: 223 - 234, 1997. J.

[10] Devooght. Dynamic Reliability. Springer, 1997.

[11] W. Lair, R. Ziani, S. Mercier, and M. Roussignol. Advances in Safety, Reliability and Risk Management, ESREL 2011, CRC Press, 885-892, 2011 